\title{
La vinculación una función sustantiva en la universidad del Ecuador y su impacto en la sociedad
}

\author{
Muñoz Atiaga Diego Rafael \\ drmunoz1@espe.edu.ec \\ https://orcid.org/0000-0003-0410-7190 \\ Torres Espín Enma Mariely \\ emtorres1@espe.edu.ec \\ https://orcid.org/0000-0003-0238-192X \\ Chuqui Chacha Jeniffer Jazmín \\ jjchuqui@espe.edu.ec \\ Cabezas Mejía Edison Damian \\ https://orcid.org/0000-0003-2726-1211 \\ edcabezas@espe.edu.ec \\ Universidad de las Fuerzas Armadas ESPE \\ Latacunga - Ecuador
}

\section{RESUMEN}

Es necesario que la universidad debe abrir sus puertas, las Instituciones de Educación Superior (IES) deben reconocer las necesidades de los sectores sociales y productivos y de esta manera superar la concepción que la vinculación es una de las funciones sustantivas de la educación superior; pero abrirlas siempre en cuanto la universidad centre su atención en el desarrollo de la ciencia, la tecnología y la innovación, como elementos básicos en el quehacer universitario; asumiendo ese liderazgo indelegable que le corresponde y sea participe del desarrollo económico, social y político; en este sentido mientras las IES del Ecuador no encuentren cabida en las actividades productivas, y que contribuyan de ésta manera al crecimiento de la economía nacional, el país no avanzará. Por ello la universidad no puede permitir el distanciamiento de la docencia y la investigación; este resquebrajamiento produce una vinculación deficiente, lejos de brindar soluciones a la comunidad; ahí radica la responsabilidad de las IES que debe orientarse a liderar el desarrollo del país a través de la mejora cualitativa de sus procesos. Para evidenciar lo mencionado se desarrolló una investigación de revisión descriptiva, a través de un diseño de tipo documental, utilizando métodos como: el histórico lógico, el 
comparativo, analítico - sintético; caminos que permitieron identificar la importancia de la vinculación como una función sustantiva en las Instituciones de Educación superior en el Ecuador.

Palabras claves: vinculación; función sustantiva; comunidad, sector productivo; instituciones educación superior 


\title{
Linking a substantive function in the university of Ecuador and its impact on society
}

\begin{abstract}
It is necessary that the university should open its doors, the Higher Education Institutions (IES) should recognize the needs of the social and productive sectors and in this way overcome the conception that bonding is one of the substantive functions of higher education; but always open them as soon as the university focuses its attention on the development of science, technology and innovation, as basic elements in university work; assuming that non-delegable leadership that corresponds to him and is a participant in the economic, social and political development; In this sense, as long as the Higher Education Institutions of Ecuador do not find a place in productive activities, and that they contribute in this way to the growth of the national economy, the country will not advance. For this reason, the university cannot allow the distancing of teaching and research; this breakdown produces a deficient relationship, far from providing solutions to the community; therein lies the responsibility of the IES, which should be oriented to lead the development of the country through the qualitative improvement of their processes. To demonstrate the aforementioned, a descriptive review investigation was developed, through a documentary-type design, using methods such as: logical historical, comparative, analytical - synthetic; Paths that allowed identifying the importance of bonding as a substantive function in Higher Education Institutions in Ecuador.
\end{abstract}

Keywords: bonding; noun function; community, productive sector; higher education institutions

Artículo recibido: 10. Junio. 2021 Aceptado para publicación: 16. Julio. 2021 Correspondencia: drmunoz1@espe.edu.ec Conflictos de Interés: Ninguna que declarar 


\section{INTRODUCCIÓN}

Es evidente que la educación superior cumple un rol estratégico en el proyecto de desarrollo económico, social y político, en el que se encuentra comprometido el país. Es necesario tener una Universidad que tenga la capacidad de formar nuevas generaciones, para que asuman de forma responsable y competente los múltiples compromisos que demanda la construcción de una nueva sociedad. La realidad evidencia que la actual formación universitaria no responde, ni cuantitativamente, ni cualitativamente a la necesidades y exigencias de la sociedad ecuatoriana.

En este sentido se deben abordar diferentes contextos, para el análisis; la naturaleza de la relación ciencia, tecnología y sociedad y las relaciones de la Universidad con la sociedad Este último contexto es el motivo de la presente investigación, por ello prioritario que la Universidad ecuatoriana construya una visión futurista, que permita al sistema de educación superior, encaminar sus esfuerzos en el desarrollo y consolidación de programas de vinculación estratégicos que permitan alcanzar objetivos que se fije la sociedad. Tal como lo refiere (Misas A. y Oviedo L. 2004). En términos generales las funciones principales de la educación superior, ésta asume diferentes tareas sociales a través de la docencia, investigación y vinculación, formando profesionales idóneos y de excelencia, desarrollando conocimiento científico, explorando sistemáticamente soluciones para los problemas que presenta el entorno y participando en forma directa en la comunidad en los análisis y en las diferentes acciones que estén orientadas a resolver problemas urgentes y con el fin de mejorar las condiciones de vida de la sociedad ecuatoriana.

La vinculación con la sociedad es uno de los tres pilares sustantivos de la Universidad, sin embargo en la actualidad se evidencia que es el pilar menos atendido en las Instituciones de Educación Superior (IES); tal vez porque no terminan por integrarse la docencia con la investigación, esto produce una brecha cada vez más grande entre Sociedad con la Universidad; este sentido la vinculación se entiende, en la mayoría de las IES, como la realización de acciones en diversos géneros, caracterizadas por ubicarse fuera de las actividades académicas formales en las IES; a pesar de que en algunos casos constituyen apoyo importante a la docencia o a la investigación, por otro lado cuando están orientadas a la comunidad del entorno de la Universidad. (Brito, Quezada, y Arzola 2018). 
En relación a lo mencionado. Las universidades deben cumplir con su misión fundamentada en los tres componentes: la educación, la investigación y la vinculación con la sociedad; componentes que se interrelacionan el uno con el otro, desarrollando una comunicación de doble dirección entre ellos. En este sentido de acuerdo a la afirmación de diversos autores que afirman que la educación debe fundamentarse en la investigación y esta debe desarrollarse en relación de las múltiples necesidades que produce la sociedad. Otro elemento que aporta y agranda la brecha entre la Universidad y la Sociedad; es que la Universidad vive en un mundo apartado de la realidad y realiza la investigación en laboratorios cerrados, olvidándose de las necesidades reales de la sociedad. Ahí radica el problema de las universidades que deben moverse en la dirección que les permita aportar con soluciones y asumir su nuevo rol de participación en el desarrollo económico, social, político.

Por ello entender el rol de la universidad del siglo XXI, es uno de los aspectos que se investigan en la actualidad, en tal razón se plantea la presente investigación en identificar la importancia de la Vinculación como una función sustantiva en la Universidad del Ecuador.

\section{La vinculación a través de los tiempos.}

Las universidades clasistas se oponían a la creación de puentes entre la sociedad y la universidad, sino hasta la mitad del siglo XIX, en los Estados Unidos que se introdujo políticas de relacionar el quehacer universitario con las necesidades e intereses del desarrollo nacional. En esta línea Campos y Sánchez 2012, plantean que la universidad latinoamericana tiene una tarea pendiente con sus respectivos entornos productivos. En el Ecuador las primeras iniciativas relacionadas con la vinculación aparecen a inicios del siglo XX, lideradas por la Universidad Central de Ecuador, en donde un minúsculo grupo de docentes desarrollaban intermitentes conferencias en diferentes disciplinas dentro y fuera de la Universidad; destinados a operarios urbanos de la ciudad de Quito, posterior a estas manifestaciones en 1940 se publicó el libro "América cursos de extensión cultural"(Espinosa 2021).

La Reforma Universitaria de 1918, también conocida como Reforma Universitaria de Córdova, marcó un antes y un después en la historia del quehacer universitario; pues se incorpora de manera formal en la universidad, la extensión universitaria, cuyo objetivo fue "poner el saber universitario al servicio de la sociedad y hacer sus problemas tema 
prioritario de sus preocupaciones" a partir del momento el concepto de extensión universitaria ha ido evolucionando, hasta convertirse en un pilar de la educación superior, conocida como vinculación con la colectividad y en la actualidad en el Ecuador como vinculación con la sociedad término utilizado a partir de la Constitución de 1998 y luego en la Ley de Educación Superior del año 2000. (Barreno Salinas et al. 2018) La vinculación en el Ecuador en la década anterior ha tenido avances importantes, pero no los suficientes, en este sentido se deben continuar planteando la necesidad de obtener resultados pertinentes y cualitativamente superiores.

\section{Sustento legal de la vinculación}

La vinculación con la sociedad se encuentra normada de acuerdo a los tratados constitucionales que rigen el accionar de la IES en el Ecuador; por ejemplo, mencionar el Art. 13 de la Ley Orgánica de Educación Superior- LOES determina que: Funciones del Sistema de Educación Superior. Son funciones del Sistema de Educación Superior (...) a) Garantizar el derecho a la educación superior mediante la docencia, la investigación y su vinculación con la sociedad, y asegurar crecientes niveles de calidad, excelencia académica y pertinencia".

Asimismo, el artículo 117 de la LOES, establece como una de las funciones sustantivas de la Universidad y Escuelas Politécnicas, lo siguiente: docencia, investigación y vinculación con la sociedad. En relación el artículo 125, de la LOES, en relación a los programas y cursos de vinculación con la sociedad, determina que las instituciones del Sistema de Educación Superior realizaran programas y cursos de vinculación con la sociedad guiados por el personal académico.

Se observa que la vinculación esta planamente respaldada por organismos que regulan la educación superior en el país; organismos como la Ley Orgánica de Educación Superior (LOES), Red Ecuatoriana Universitaria de Vinculación con la Colectividad (REUVIC); Reglamento de Régimen Académico en el Sistema de Educación Superior (RRA) y la Red Ecuatoriana Universidades y Escuelas Politécnicas para la Investigación y Posgrados (REDU), entre otras. (Ley Orgánica Educación Superior (LOES) 2010).

En relación a los organismos que impulsan el quehacer universitario, estos propician el acercamiento, la comunicación, las relaciones entre las IES, de ahí su finalidad de viabilizar a la vinculación con la sociedad, como un eje de articulación con la docencia y la investigación Asimismo promueven programas, proyectos y el desarrollo de 
actividades académicas interinstitucionales, también regulan la gestión académica formativa en todos los niveles de formación y modalidades de aprendizaje en la educación superior del país, con el fin de mejorar la investigación, la formación académica y profesional, la investigación y la vinculación con la sociedad.(Barreno Salinas et al. 2018) A pesar de acciones desarrolladas por los organismos y de acuerdo a los contextos legales que la amparan; la vinculación con la IES se la conceptualiza de manera difusa cayendo en la interpretación del concepto de vinculación con la sociedad, entendiéndose en algunas ocasiones como equivalente a extensión universitaria, limitando el accionar de las IES con el sector productivo, entendiéndose con las industrias, empresas y comercios; por ello estas malas interpretaciones han desembocado en diferentes formas de entender a la vinculación:

- Considerar que la vinculación tiene un contenido básicamente económico; en este caso la vinculación se ve básicamente como venta de servicios.

- Considerar que la vinculación se resuelve exclusivamente mediante un acercamiento físico entre universidad con la sociedad; esta es una visión fisicalista, que también está fuertemente asociada a una visión asistencial.

- Considerar que la vinculación es una nueva función académica de las universidades; esta perspectiva sostiene que tanto la docencia, como la investigación y la vinculación con la sociedad son funciones sustantivas que articulan el quehacer universitario. (Campos y Sánchez 2012)

Estas diferentes interpretaciones de la vinculación, ha provocado una confusión del concepto de vinculación con la sociedad, entendiéndose en múltiples ocasiones como una forma de filantropía hasta un equivalente de extensión universitaria. En tal circunstancia más allá de las diferentes interpretaciones, la Universidad debe tener claro la responsabilidad social universitaria que debe contribuir a clarificar y fortalecer la relación Sociedad - Universidad, a través de acciones ejercida desde una perspectiva ética y moral, contribuyendo a esa responsabilidad que deben ejercer todos los protagonistas del quehacer universitario; por ello la vinculación será analizada desde sus diferentes formas:

\section{La vinculación con la comunidad como elemento de desarrollo}

El Consejo de Evaluación, Acreditación y Aseguramiento de la Calidad de la Educación Superior (CEAACES) en el año 2010 expidió una reformo a la Ley de Vinculación Social, en el cual obliga a todas las universidades públicas y privadas realizar proyectos de 
vinculación social. En este sentido a pesar de esta ley, la vinculación no ha tenido un protagonismo determinante en el claustro universitario ecuatoriano; razones pueden ser varias como por ejemplo esa mentalidad que se mantiene de asistencialismo, dependencia y mentalidad de invalidez que muestran las comunidades; eso debe cambiar debe transformarse por dinámicas de estudio, de análisis y dialogo que posibiliten transformaciones para alcanzar un aprendizajes significativo; solo así se podrá erradicar la pobreza.(Tello 2017)

En este contexto se manifiesta que las actividades de vinculación puede actuar como una estrategia de desarrollo, debido a que esta puede promover el desarrollo de nuevas líneas y sub líneas de investigación y posibles cambios en la oferta académica, producto de las realidad y demandas sociales; también se puede conformar mesas de trabajo para analizar las problemáticas que afectan a la nación; a través de estas y otras actividades la universidad recuperará esa imagen institucional, ese liderazgo indelegable que debe ejercer la universidad ecuatoriana. En este contexto la Universidad ecuatoriana debe a partir de sus necesidades adoptar su propio modelo de vinculación que se adapte al contexto, tomando en cuenta las características, las culturas, las potencialidades, las debilidades, y oportunidades; de esta manera se fortalecerá la imagen institucional de la Universidad. (Indacochea, et.at. 2018)

\section{La vinculación con la sociedad y los sectores productivos}

Un elemento decisivo para que exista una relación entre la Universidad y los sectores productivos, es la preparación de su población en el ámbito educativo, ligada al desarrollo del conocimiento y a la ciencia; esta razón ha creado en los países industrializados una marcada diferencia con los países no industrializados o en vías de desarrollo, han descuidado el desarrollo educativo, produciendo elevados niveles de desigualdad económica y social. A lo manifestado los países industrializados mantiene una filosofía de eficacia y calidad de la universidad y está directamente relacionada con la sociedad; en cambio en América Latina, la Universidad y el Sector Productivo (SP) han evitado esta relación, debido a que la Universidad se mantiene a la expectativa del cumplimiento de sus funciones sustantivas (docencia, investigación y vinculación), y el SP mantiene paradigmas de desconfianza y prejuicios, respecto al rigor académico en los universitarios, ubicándolos de manera lejana en temas relacionados con la producción.(Alonso, Cú, y Aragón 2014) 
Es necesario que la universidad debe abrir sus puertas, las IES deben reconocer las necesidades de los sectores sociales y productivos y de esta manera superar la concepción que la vinculación es un camino de una sola vía, al contrario deben establecer y buscar estrategias que conduzcan al planteamiento de programas, proyectos en donde la Universidad y el sector productivo alcancen beneficios valiosos; pero para llegar a esta realidad debe la Universidad debe re-direccionar las funciones sustantivas, como docencia, investigación y vinculación para ello la Universidad debe preocuparse de firmar convenios, contratos de asesoría con el sector productivo del país, para de esta manera tenerle al sector productivo íntimamente relacionado con la academia. (Indacochea, et.at. 2018)

Asimismo, mencionar que la vinculación con el sector productivo, es válida siempre y cuando se la realice con la estructura industrial brindando atención a los problemas tecnológicos del sector productivo; en otras palabras, transferencia de tecnología; esta trasferencia producirá confianza en el empresario y estará dispuesto a invertir en proyectos de investigación que sean liderados por el claustro universitario; consiguiendo un beneficio mutuo empresa - universidad. Esta premisa conlleva al personal de la Universidad que debe estar en constante preparación a lo largo de toda la vida para dar solución a las necesidades y requerimientos que están en constante cambio.

Realizando un recorrido por diferentes motores de búsqueda se identifican algunas definiciones que permitirán entender esta función sustantiva denominada vinculación para luego estas definiciones ser analizadas por los autores:

(Duque, Reyes, y Morocho 2018) plantean que la vinculación se convierte en una herramienta de enseñanza que permite a los docentes lograr integrar los saberes que el estudiante va adquiriendo durante su vida estudiantil. Además, que el docente se ve en el reto del continuo aprendizaje, debido a que las realidades a las que se enfrentarán son variables y cambiantes.

En el contexto (Simbaña 2018) afirma que vinculación es la función sustantiva que impulsa la interacción de los actores universitarios con los actores sociales para transformar la realidad, a través de acciones que articulan el quehacer de la vida universitaria; que transcienda en el ámbito interno y externo de la IES. En tal circunstancia la vinculación es una herramienta metodológica participativa, consiente y 
recíproca y de compromiso que permite el intercambio y la innovación de los saberes y cocimiento.

En el tenor de las ideas, bajo esta perspectiva, (Simbaña y Correa 2018) afirman que se puede entender a la vinculación como el anuncio de un nuevo estadio de la extensión universitaria, como el resultado de un proceso sistémico realizado en los últimos tiempos en nuestro país, donde se afirma que la función a la que hacemos referencia, ha dejado de ser tal cosa para evolucionar a un aspecto más avanzado denominado Vinculación con la Sociedad.

Por su parte (Polaino y Romillo 2017) mencionan que la vinculación; es una de las funciones sustantivas de la educación superior que, de conjunto con la formación académica y la investigación, tiene el propósito de solucionar problemas de la comunidad, a través de proyectos, prácticas pre-profesionales, capacitación y otros servicios profesionales, que posibilitan la comunicación y la interacción efectiva de la universidad con su entorno, así como la integración de la teoría con la práctica del quehacer universitario en función del desarrollo social sostenible.

El objetivo planteado para la presente investigación es determinar la importancia de la Vinculación como una función sustantiva en la Universidad del Ecuador, con base en el análisis de investigaciones publicadas en bases de datos relevantes.

\section{ESTRATEGIAS METODOLÓGICAS O MATERIALES Y MÉTODOS}

El propósito de desarrollar el presente documento de revisión se planteó la aplicación de diferentes métodos de investigación como el análisis y síntesis de diferentes fuentes documentales, en donde se evidencia contenidos relevantes relacionados al objeto de estudio que es la vinculación con la sociedad; asimismo se utilizó métodos como el histórico lógico en la fundamentación teórica y en la parte final del artículo de revisión se aplicó el método comparativo, que permitió desarrollar comparaciones y discusiones a partir de los resultados y hallazgos encontrados en las diferentes investigaciones que servirán de insumos en el presente trabajo.

El diseño investigativo es descriptivo por su esencia del tipo de artículo de revisión; en el cual se presenta en un primer momento una conceptualización y fundamentación teórica en torno a la vinculación con la sociedad; el trabajo descriptivo se lo realizó a partir de quince investigaciones consultadas en importantes bases de datos indexadas como Latindex, Redlic, Scielo Revlatinoamericana, Dialnet; entre otras. Además se 
caracterizaron factores internos y externos que influyen en el desarrollo de esta función sustantiva de la Universidad en el Ecuador y en un tercer momento final se incluyen las conclusiones sobre la importancia de integrar la estructura organizacional de la sociedad con la Universidad.

\section{RESULTADOS Y DISCUSIÓN}

Una vez fundamentados los elementos teóricos de la función sustantiva de la Educación Superior como es la Vinculación se procedió a discutir los resultados de las diferentes investigaciones relacionadas con la variable, con la finalidad de alcanzar el objetivo de la investigación:

Sobre los aspectos relacionados con los procesos de gestión (Cedeño et al. 2018) concluye que las universidades y la vinculación con la sociedad, necesitan además de que sus procesos de gestión sean más efectivos; deben procurar que sus acciones integren a las entidades del gobierno, funcionarios y protagonistas de los sectores productivos, la tecnología, la ciencia, entre otros; por lo cual el enfoque se convierte en complejo permitiendo una estructura organizacional que, a la vez, se mire a la docencia y la investigación como elementos fundamentales, para que la vinculación tenga un efecto positivo en la sociedad como parte de la solución.

Conclusión que se relaciona con los datos de (Indacochea, 2018) quien considera que la universidad se fundamenta en la mejora continua, en la calidad de sus procesos de gestión de docencia, investigación y vinculación; en la capacidad que tenga para repensar e innovar su aporte ante una sociedad cambiante; las IES debe dar respuestas estratégicas a través de la vinculación para el desarrollo lo que permite generar actividades en la que los estudiante puedan aprender juntos, es decir los participantes sean estos los educandos como los beneficiarios de determinado proyecto.

En referencia a la vinculación como eje de desarrollo académico (Simbaña y Correa 2018) concluye que el avance incontenible de la ciencia y la tecnología demanda de la casona de estudios mayores niveles de calidad y pertinencia en la formación de profesionales. Potencializar su trascendencia académica y política, exige a la universidad actual, analizar sus experiencias en este campo, contextualizarlas a la realidad de los pueblos de Ecuador, para proyectarlas en el siglo XXI, desde una perspectiva trasformadora, holística e integral, como instrumento que garantice un cambio cualitativo en la educación superior. Estos resultados coinciden con los preconizado por (Chávez 
2016) quien manifiesta que debe superarse las viejas limitaciones que tiene la universidad con respecto a los actores sociales y productivos. Esto ha ocasionado que la universidad no oriente adecuadamente sus proyectos y líneas de investigación a la resolución de problemas de alto impacto en la vida de los ciudadanos, así como de las empresas que demandan sus servicios, esto por consecuencia de un distanciamiento de la docencia y la investigación que no han transcendido en la universidad ecuatoriana; y las pocas iniciativas han quedado en la sombra, sin la posibilidad de convertirse en algo que día a día debe desarrollarse.

(Barreno Salinas et al. 2018) El papel de las IES ante las demandas de colaboración con los procesos del desarrollo socioeconómico y cultural no sólo con la sociedad sino entre ellas mismas ha cambiado de una visión reduccionista y limitada, a una de mayor proyección con un amplio espectro de posibilidades acorde a las tendencias socioeconómicas mundiales. En el contexto de la educación superior ecuatoriana la vinculación con la sociedad se ha convertido en un factor de interacción e intervención estratégico en el desarrollo sociopolítico y cultural tanto local, como regional y nacional haciendo posible una formación de alta calidad del Sistema de Educación Superior ecuatoriano. Esta afirmación no concuerdan con lo manifestado por (Tello 2017) quien concluye en su investigación que la vinculación en el Ecuador no ha tenido un protagonismo determinante en el claustro universitario ecuatoriano; razones pueden ser varias como por ejemplo esa mentalidad que se mantiene de asistencialismo, dependencia y mentalidad de invalidez que muestran las comunidades; eso debe cambiar debe transformarse por dinámicas de estudio, de análisis y dialogo que posibiliten transformaciones para alcanzar un aprendizajes significativo; solo así se podrá erradicar la pobreza.

En este orden de ideas (Barreno Salinas et al. 2018) concuerdan con (Tello 2017) en coincidir con varios autores en el fracaso de la vinculación de las instituciones de educación superior (IES) con la sociedad, en tanto las primeras no siempre guardan el vínculo y manifiesta que la vinculación entre la universidad latinoamericana, de manera general, y sus respectivos entornos productivos es una tarea que continua pendiente, a pesar de ciertas excepciones. En el caso de Ecuador, aunque en la última década las IES han tenido avances, pero muy lentos en este sentido, se continúa planteando la necesidad de obtener resultados pertinentes y cualitativamente superiores. 
A vista de la realidad mientras las Instituciones de Educación Superior no encuentren cabida en las actividades productivas, y que contribuyan de esta manera al crecimiento de la economía nacional, el país no avanzará, y puntualizar que ambas instituciones deben asumir una responsabilidad y compromiso compartido. En relación a lo mencionado (Alonso et al. 2014) que mientras los países industrializados mantienen una filosofía de eficacia y calidad de la universidad y está directamente relacionada con la sociedad; en cambio en América Latina, la Universidad y el Sector Productivo (SP) han evitado esta relación, debido a que la Universidad se mantiene a la expectativa del cumplimiento de sus funciones sustantivas (docencia, investigación y vinculación), y el SP mantiene paradigmas de desconfianza y prejuicios, respecto al rigor académico en los universitarios, ubicándolos de manera lejana en temas relacionados con la producción.

En concordancia a lo mencionado (Indacochea, 2018) afirma elementos relacionados al estado de la vinculación de las IES con el sector productivo, en donde manifiesta que estas relaciones deben estar apoyados por los sectores estatales considerando la importancia de la ciencia, la tecnología y la innovación, como elementos básicos en el quehacer universitario, que deben desarrollar los tutores e investigadores; a partir de ello el sector productivo establecerá relaciones de vinculación con la casona universitaria, solo ahí se creará una estrategia general de intervención que se permita plasmar en muchas prácticas educativas hacia las necesidades de la realidad productiva.

En relación a la vinculación con el sector productivo en la región, México es una excepción en donde (Alonso et al. 2014) concluye: mientras exista la comunicación cercana y continua entre universidades y empresas, y la educación siga siendo en México factor de movilidad social y los cuadros profesionales formados en las Instituciones de Educación Superior, encuentren cabida en las actividades productivas, contribuirán de ésta manera al crecimiento de la economía nacional, ambas instituciones deben seguir asumiendo una responsabilidad y compromiso compartido. Contrario a esta realidad en el Ecuador (Chávez 2016) menciona que en las organizaciones empresariales, la dependencia de la tecnología importada seguirá ocasionado un divorcio con la universidad en cuanto a soluciones a partir de elementos nacionales. En ese contexto, las universidades han dejado de lado su rol protagónico frente a otras universidades extranjeras. Por tanto, lo primero a superar en la actual es la deslegitimación de la 
universidad como prestadora de servicios empresariales y tecnológicos y superar la desconfianza entre empresarios e investigadores

\section{CONCLUSIÓN O CONSIDERACIONES FINALES}

Producto del análisis y discusiones de las diferentes investigaciones que abordan la vinculación como elemento sustantivo de las IES, se propone las siguientes consideraciones finales:

Es evidente que en las últimas décadas, se han impulsado diversas políticas de gobierno, por parte de los entes rectores de las IES, tendientes a fortalecer una de las funciones sustantivas de la Educación Superior, como es la vinculación; en la actualidad la sociedad demanda mecanismos que permitan la relación entre Universidad y los sectores sociales y productivos; por ello es prioritario trabajar de manera planificada y en equipo, con los actores sociales para trasformar la realidad, a través de acciones que articulen el quehacer de la vida universitaria, que el producto de la vinculación trascienda en el ámbito interno y externo de las Instituciones de Educación Superior.

La universidad debe responde a los requerimientos actuales de la política social incorporando en sus programas de estudios, asignaturas que permitan brindar soluciones a través del trabajo social y desarrollo comunitario. La universidad no puede permitir el distanciamiento de la docencia y la investigación; este resquebrajamiento produce una vinculación insipiente, lejos de brindar soluciones a la comunidad; la responsabilidad de las IES debe orientarse a liderar el desarrollo del país a través de la mejora cualitativa de sus procesos. Estas realidades nos invitan a desaprender y reaprender los procesos de mejoramiento ante una sociedad tan cambiante, permitiendo de esta manera un intercambio de los saberes y conocimientos entre la Universidad y la Sociedad.

Es evidente que las IES no encuentran cabida en las actividades productivas y poco o nada contribuyen a las actividades productivas del Ecuador, esta realidad no cambiara mientras exista una escasa labor investigadora, consecuencia de una elevada proporción del presupuesto de la IES destinado a la docencia y a la carga administrativa; a pesar de estos limitantes la universidad tiene que formar no solo profesionales que formen a estudiantes, sino también formar profesionales que tengan la capacidad de desarrollar investigación y formular respuestas a las grandes problemáticas de la sociedad. 


\section{LISTA DE REFERENCIAS}

Alonso, Cú, y Aragón. 2014. «LA VINCULACIÓN UNIVERSIDAD SECTOR PRODUCTIVO». Recuperado 17 de junio de 2021 (http://www.quadernsdigitals.net/datos/hemeroteca/r_1/nr_751/a_10212/10212.h tml).

Barreno Salinas, Mercedes, Zoila Barreno Salinas, Adriana Carolina Olmedo Valencia, Mercedes Barreno Salinas, Zoila Barreno Salinas, y Adriana Carolina Olmedo Valencia. 2018. «La educación superior y su vinculación con la sociedad: referentes esenciales para un cambio». Revista Universidad y Sociedad 10(3):4045.

Brito, Quezada, y Arzola. 2018. LaUniversidad-y-LaVinculacionConLaSociedad.pdf. primera. Machala:

UTMACH. http://repositorio.utmachala.edu.ec/bitstream/48000/12492/1/LaUniversidad-yLaVinculacionConLaSociedad.pdf

Cedeño, Vélez, Ostaíza, y Solórzano. 2018. «Vinculación con la sociedad: un desafío posible desde el altruismo, el compromiso y la organización.» REVISTA SAN GREGORIO http://revista.sangregorio.edu.ec/index.php/REVISTASANGREGORIO/article/v iew/593/7-ROSA

Chávez. 2016. «UNIVERSIDAD, VINCULACIÓN CON LA SOCIEDAD Y PROCESOS DE MEJORAMIENTO». Quipukamayoc 24:15. doi: 10.15381/quipu.v24i45.12456.

https://www.researchgate.net/publication/319660994_UNIVERSIDAD_VINCU LACION_CON_LA_SOCIEDAD_Y_PROCESOS_DE_MEJORAMIENTO/cit ation/download

Duque, $\quad$ Reyes, $\quad$ y $\quad$ Morocho. 2018. «el_proyecto_universitario_de_vinculacion_con_la_sociedad.pdf». https://www.pedagogia.edu.ec/public/docs/Comision_10/el_proyecto_universita rio_de_vinculacion_con_la_sociedad.pdf

Espinosa. 2021. «Vista de Vinculación con la sociedad». Revista Arquitectura y Sociedad 1:13. https://revistadigital.uce.edu.ec/index.php/arquitectura/article/view/2989 
Ley Orgánica Educación Superior (LOES). 2010. Ley Orgánica Educación Superior (LOES).

https://siteal.iiep.unesco.org/sites/default/files/sit_accion_files/ec_6011.pdf

Misas A., Gabriel, y Mónica Oviedo L. 2004. La educación superior en Colombia: análisis y estrategias para su desarrollo. 1. ed. Bogotá, D.C., Colombia: Universidad Nacional de Colombia. https://www.urosario.edu.co/Subsitio/Forosde-Reforma-a-la-Educacion-Superior/Documentos/GMA---La-EducacionSuperior-en-Colombia.pdf

Montenegro, Duarte, y José Enrique. 2008. «Vinculación universidad-sector productivo: Una visión para el desarrollo tecnológico de las organizaciones». 17. https://www.redalyc.org/pdf/4655/465545879006.pdf

Polaino, Cecilia J., y Antonio de J. Romillo. 2017. «Vinculación con la Sociedad en la Universidad de Otavalo, Ecuador». Formación universitaria 10(3):21-30. doi: 10.4067/S0718-50062017000300004.

Rueda, Iván, Byron Acosta, y Fabián Cueva. 2020. «LAS UNIVERSIDADES Y SUS PRÁCTICAS DE VINCULACIÓN CON LA SOCIEDAD». Educação \& Sociedade 41. doi:

10.1590/ES.218154. https://www.scielo.br/j/es/a/3jGkPBzQG9GJ7NVj8XnY5tB/?lang=es

Simbaña, y Correa. 2018. «La vinculación con la sociedad en el Ecuador: Reflexiones sobre su itinerario curricular». Revista Vínculos 2(1). doi: 10.24133/rvespe.v2i1.563.

https://journal.espe.edu.ec/ojs/index.php/vinculos/article/view/563

Simbaña, Ph D. Héctor. 2018. «LA VINCULACIÓN CON LA SOCIEDAD.» 18. https://www.caces.gob.ec/wp-content/uploads/2020/02/HECTORSIMBANA.pdf

Tello, Augusta Carolina Rivas. 2017. «EFECTOS DEL MODELO DE VINCULACIÓN CON LA SOCIEDAD DE LA FACULTAD DE COMUNICACIÓN SOCIAL DE LA UNIVERSIDAD CENTRAL DEL ECUADOR, DESDE LA PERSPECTIVA DEL ENTORNO DE APRENDIZAJE». Revista de Ciencias de Seguridad y Defensa 2(7):10. HTTPS://JOURNAL.ESPE.EDU.EC/OJS/INDEX.PHP/REVISTASEGURIDAD-DEFENSA/ARTICLE/VIEW/2099 